\title{
Umidificação do trato respiratório em pacientes sob ventilação mecânica: uma revisão bibliográfica
}

\author{
Humidification of the respiratory tract in patients with mechanical syndrome: a literature \\ review
}

Kamilla Soares dos Reis ${ }^{1}$, Geovane Rossone Reis ${ }^{2}$, Anny Pires de Freitas Rossone $^{3}$

\section{RESUMO}

Introdução: A incidência de pneumonia associada à ventilação mecânica é relativamente alta nas unidades de terapia intensiva, e os cuidados intensivos, bem como, o manejo com os circuitos do ventilador mecânico podem aumentar ou reduzir esta incidência. Pacientes em ventilação mecânica necessitam de interfaces para aquecer e umedecer o ar inspirado através das próteses respiratórias. Os dispositivos utilizados para a umidificação do ar inspirado - umidificador aquecido e trocadores de calor e umidade - podem ter alguma associação com este tipo de infecção. Objetivo: Esclarecer, através do levantamento do resultado das principais pesquisas, as melhores formas de umidificar as vias aéreas de pacientes sob ventilação mecânica. Metodologia: Foi realizado uma revisão da literatura, onde foram coletado os resultados dos principais estudos contidos no Portal de Periódicos da CAPES, na Scielo e no LILACS Discussão: A maioria dos artigos esclare que o filtro trocador de calor e umidade gera uma umidicação mais fisiológica ao trato respiratório, porém algumas pesquisas não comprovam maior eficiência entre o filtro e o umidificados aquecido. Conclusão: A umidificação das vias aéreas é crucial para a condução de pacientes sob ventilação mecânica, porém não há um consenso sobre qual é o melhor método de umidificação.

Descritores: Respiração artificial, Administração por inalação, Trato respiratório.

\section{ABSTRACT}

Introduction: The incidence of ventilator-associated pneumonia is relatively high in intensive care units, and intensive care, as well as management with mechanical ventilator circuits, may increase or decrease this incidence. Patients on mechanical ventilation require interfaces to warm and moisten the inspired air through the respiratory prosthesis. The devices used for humidifying the inspired air - heated humidifier and heat and moisture exchangers - may have some association with this type of infection. Objective: To clarify, by surveying the results of the main research, the best ways to humidify the airways of patients under mechanical ventilation. Methodology: A review of the literature was carried out, where the results of the main studies contained in the Portal of Periodicals of CAPES, SciELO and LILACS were collected. Discussion: Most articles indicate that the heat exchanger and humidity filter generates a more physiological humidification to the respiratory tract, but some research does not prove a greater efficiency between the filter and the heated humidifier. Conclusion: Humidification of the airways is crucial for the management of mechanically ventilated patients, but there is no consensus as to the best humidification method.

Descriptors: Artificial Respiration, Administration, Inhalation, Respiratory system
${ }^{1}$ Fisioterapeuta (UnirG-TO), pós-graduanda em terapia intensiva pela UnirG.

${ }^{2}$ Fisioterapeuta (UBM-RJ),

especialista em ventilação mecânica, mestre em gestão de políticas públicas pela Universidade Federal do Tocantins.

${ }^{3}$ Fisioterapeuta (UnirG-TO) especialista em saúde pública pelo IEP-TO.

\section{Endereço para}

correspondência:

Universidade Gurupi, Av. Rio de Janeiro no 1585, Centro 77403-090, Gurupi - TO, Telefone: (63) 9.9996 .7555

Email: 


\section{INTRODUÇAOO}

As vias aéreas que vão desde o nariz aos bronquíolos terminais e sua superfície necessitam de três funções que são: umedecer, filtrar e aquecer 0 ar inspirado. A umidade acontece através de uma camada de muco que recobre toda a região interna da via. Este muco é secretado, em parte, por células caliciformes que se originam na camada de revestimento epitelial, e em parte, por glândulas presentes na submucosa ${ }^{1}$.

Este tem também como papel primordial, aprisionar pequenas partículas provindas do ar inspirado, impedindo assim que cheguem aos alvéolos completando a função de filtração que também se dá pela presença de pêlos na entrada das narinas, e conseqüentemente retendo grandes partículas externas. Caso alguma partícula passe, existirá o epitélio ciliado o qual atuará em batimentos ciliares impulsionando a partícula juntamente com o muco em direção a faringe, onde o muco será deglutido ou expectorado. Logo em seguida, há o aquecimento do ar, através dos septos e cornetos nasais, onde as áreas de contato são irregulares e extremamente irrigadas, assim o ar será turbilhonado e aquecido².

De acordo com Safar e Caroline (1982) ${ }^{3}$, o tubo endotraqueal salta esta fase fisiológica natural, impedindo através do próprio tubo a seqüência básica da respiração, e reduzindo assim, as defesas nasais e pulmonares e aumentando o risco de lesão na mucosa traqueobrônquica por conseqüência do ar seco inspirado.

Assim, com a diminuição das defesas biológicas, fica em aberto um vasto e facilitado caminho para as infecções pulmonares se alojarem. Sendo que este tipo de infecção já ocupa a segunda posição entre as infecções hospitalares nos Estados Unidos ${ }^{4}$.

O III Consenso de Ventilação Mecânica, $(2007)^{5}$ dita que a Pneumonia Associada à Ventilação Mecânica (PAVM) está diretamente ligada aos altos índices de morbimortalidade em pacientes imunossuprimidos e transplantados.

Por isso, há a necessidade de dispositivos de respiração artificial que mais se assemelhem à respiração humana e que simulem as suas funções de umedecer e aquecer, como o Umidificador Aquecido e os Trocadores de Calor e Umidade (HMEsHeat and Moisture Exchangers). Junior e Amaral (1998) ${ }^{2}$ relatam que o umidificador aquecido, umedece relativamente a $100 \%$ através do vapor de água e cai a $50 \%$ quando entra em contato direto com a mucosa das vias aéreas, além de ser regulado através de um sistema eletrônico de aquecimento. Já o HME, além de sua função primordial de umedecer e aquecer, ele também reduz a perda de água e diminui a condensação de 
água no circuito do ventilador, sendo este de baixo custo, e de fácil uso, tendo um papel de filtro microbiológico que não necessita de uma fonte de energia ${ }^{6}$.

O sistema respiratório é divido em trato superior e inferior. No trato superior, o ar inspirado é filtrado pelas vibrissas presentes na entrada do orifício nasal e por muco produzido por células caliciformes encontradas na camada de revestimento do epitélio de todo o trato respiratório, além de células ciliadas que influenciarão na propulsão do muco a orofaringe em todo o trato ${ }^{7}$.Ainda no trato superior o ar é aquecido a, aproximadamente $32^{\circ} \mathrm{C}$, e a umidade é relativamente maior que $90 \%$. O trato respiratório inferior segue com a função de igualar a temperatura do gás inspirado, com a temperatura corporal e umidade relativa a $100 \%$ e de aumentar a função de produção mucociliar ${ }^{6}$.

Esta série de fatores de proteção funciona como um mecanismo de defesa tanto mecânica quanto imunológico para os pulmões ${ }^{8}$.

Os principais mecanismos de defesa das vias aéreas e sua segmentação progressiva são a filtração aerodinâmica e o transporte mucociliar. Já os mecanismos imunológicos são compostos predominantemente, pelo sistema macrofágico e as células imunes efetoras ${ }^{9,10,11}$.

Em pacientes submetidos à ventilação Mecânica invasiva, estes mecanismos de defesa são alterados. O tubo endotraqueal causa mudanças nos mecanismos mecânicos e humorais de defesa das vias superiores ${ }^{12}$. Segundo Sarmento et al. (2006) ${ }^{6}$ a perda dos mecanismos naturais de umidificação, filtração e aquecimento deve ser rigorosamente controlada, pois a incidência de complicações pulmonares são aumentadas depois de um breve período de VM, sem sistema de umidificação e aquecimento ou quando este sistema é insuficiente. $O$ risco de lesão na mucosa traqueobrônquica por consequência do ar seco inspirado é aumentado, além de que na maioria das vezes é associado ao espessamento das secreções ${ }^{3}$, hipotermia, atelectasias e infecções pulmonares ${ }^{13}$. Mas, se o gás for corretamente aquecido e umidificado, reduzirá a desidratação sistêmica e a manter a temperatura corporal ${ }^{14}$.

Por estes motivos, é então indispensável o condicionamento dos gases inspirados, para que haja aquecimento e umidificação adequada e para assegurar a integridade das vias aéreas e correta função mucociliar ${ }^{15}$.

O condicionamento do ar pode ser realizado por umidificadores aquecidos ou por trocadores de calor e umidade ${ }^{7}$.

Para que ocorra a umidificação e o aquecimento do ar inspirado durante a implementação da ventilatória mecânica, comumente são utilizados dois dispositivos, 
sendo denominados umidificadores aquosos aquecidos (UAA) e filtros trocadores de calor e umidade (HME). Os UAAs promovem o aquecimento e umidificação do ar inspirado pela passagem do mesmo por meio de uma câmara preenchida parcialmente por água destilada aquecida, enquanto os HMEs são dispositivos adaptados entre as vias aéreas artificiais e o circuito do ventilador, sendo capazes de armazenar parte do calor e do vapor d'água proveniente do ar exalado, disponibilizando-os durante uma nova inspiração ${ }^{16}$. Os HMEs se dividem em três grandes grupos: higroscópicos, hidrofóbicos e mistos (higroscópico-hidrofóbicos) ${ }^{17}$.

Os HMEs higroscópicos são compostos por material de baixa condutividade térmica, carregado por sal higroscópico como o cloreto de lítio, magnésio ou cálcio para, dessa forma, reter e conservar a umidade. No percurso da expiração ocorre a condensação e por consequência retenção de água aquecida no elemento higroscópico. Com isso o gás inspirado, é umidificado e aquecido com a água retida no sistema. Esse HME tem melhor qualidade de umidificação, menor volume interno e menor resistência, além de possuir uma barreira microbiológica, porém não é considerado filtro ${ }^{18}$.

Os hidrofóbicos proporcionam uma área de superfície alargada por dobraduras recoberta por material que evita a passagem de água para o meio externo. Equivalente aos filtros higroscópicos o gás inspirado também é aquecido e umidificado pela a água retida na superfície interna do HME durante a expiração. São desprovidos de adequada umidificação, possibilitando a oclusão do tubo endotraqueal pela baixa umidade. Porém, são competentes filtradores de bactérias ${ }^{6,19}$. Estes filtros antibacterianos possuem condensadores de membranas com poros menores que 0.6 micrômetros de diâmetro ${ }^{13}$.

Em face a esta discussão, este trabalho visa analisar os principais estudos sobre o tema a fim de nortear, com base em evidências, a melhor condução dos pacientes em ventilação mecânica.

\section{MATERIAIS E METODOS}

Trata-se de uma revisão exploratória da literatura. Os artigos científicos para elaboração do mesmo foram selecionados nas bases de dados Portal de periódicos Capes, Centro Latino-Americano e do Caribe de Informação em Ciências da Saúde Bireme, Literatura Latino-americana e do Caribe em Ciências da Saúde - Lilacs e Scientific Electronic Library Online - SciELO, em português e inglês, utilizando os 
descritores em língua portuguesa: Respiração artificial, administração por inalação e trato respiratório. Além de livros sobre fisiologia do exercício e medicina intensiva.

Foram incluídos todos trabalhos e livros que abordassem o tema proposto.

O presente trabalho não precisou ser submetido ao comitê de ética em pesquisa, conforme a resolução 466/2012, pois não tem como participante o ser humano, em sua totalidade ou em partes, e não o envolve de forma direta ou indireta, incluindo o manejo de seus dados, informações ou materiais biológicos.

\section{DESENVOLVIMENTO}

Os filtros mistos associam um elemento higroscópico a um hidrofóbico, satisfazendo as necessidades de umidificação e calor diminuído nos hidrofóbicos e aumentando as propriedades de filtro contra bactérias no higroscópico ${ }^{6}$.

Esses dois umidificadores apresentam algumas desvantagens durante a sua utilização. Nos UAAs observa-se a produção de grande volume de condensado, aumento da incidência de pneumonia associada à ventilação, assíncrona paciente-ventilador, necessidade de energia elétrica e constante reabastecimento de água. Os HMEs durante uso prolongado (> 24h) pode promover o aumento do trabalho respiratório, devido a maior resistência oferecida à passagem do gás inspirado, além de alterações da ventilação alveolar ${ }^{16}$ e obstrução do aparelho ${ }^{18}$. Cerca de $95 \%$ dos sistemas ventilatórios são colonizados após 24 horas de uso, por isso sua troca se dá a cada 24 horas. O uso excedente do prazo recomendado desses promove a contaminação por microrganismos, tornando mais propenso a infecções respiratórias. Outros estudos, mostram que a permanência dos HMEs em 48 horas e 96 horas não alterou o seu funcionamento em termos de aquecimento, água e preservação dos gazes ventilatórios e não propiciou a colonização de bactérias ${ }^{20,21}$.

O risco de infecção e de morte hospitalar é aumentado durante a permanência na $\mathrm{UTI}^{22}$. Contudo, os riscos de se desenvolver infecção como a pneumonia nosocomial, é elevada em pacientes entubados e criticamente doentes. Considera-se pneumonia nosocomial quando não há evidencias de presença ou incubação da doença no momento da admissão na UTI23.

A pneumonia associada à ventilação mecânica (PAVM) é denominada nos estudos histológicos por acúmulo de neutrófilos na região dos bronquíolos distais e alvéolos. É a infecção adquirida na unidade de terapia intensiva (UTI) mais freqüente entre os 
pacientes submetidos a este suporte ventilatório, é caracterizado pela multiplicação de microrganismos nas vias aéreas distais ${ }^{24}$. Ocorrem 48 horas após a internação em um paciente em uso de ventilação mecânica ${ }^{25}$.

Torres e Ewig (2007) ${ }^{26}$ oferecem diretrizes na suspeita de PAVM, onde se baseiam em critérios clínicos logo citados: presença de infiltrados novos e persistentes associados a dois dos seguintes: temperatura $>38,3^{\circ} \mathrm{C}$, leucocitose ou leucopenia, ou secreção traqueobrônquica purulenta ou somente um dos citados, e desconforto respiratório agudo do adulto ou escore de infecção pulmonar clínico > 6 (varia de 0 a 12), deve-se obter para a fase inicial secreções do trato respiratório para culturas quantitativas (amostras de aspirado traqueal ou lavado bronco-alveolar), e rapidamente iniciar a antibioticoterapia e reavaliar em 48 a 72 horas.

\section{CONSIDERAÇOES FINAIS}

Independente de não existir valores significativos entre o UAA e o HME na redução da incidência de PAVM, o tipo HME é um método alternativo recomendado para a umidificação de pacientes que estejam em VM pois a umidificação e a temperatura mantida pelo mesmo tem maior semelhança com a necessidade fisiológica da respiração ${ }^{23,27}$.

\section{REFERENCIAS}

1. GUYTON, Arthur C.; HALL, John E. Tratado de fisiologia médica. 10. ed. Rio de Janeiro: Guanabara Koogan, 2002. 975 p.

2. VINCENT J. L., RELLO, J., MARSHALL, J.,SILVA, E.,ANZUETO, A.,MARTIN, C. D., MORENO, R., LIPMAN, J., GOMERSALL, C., SAKR, Y., REINHART, K. International Study of the Prevalence and Outcomes of Infection in Intensive Care Units. (Reprinted) JAMA, December 2, 2009-Vol 302, No. 21

3. RICHARDS MJ, EDWARDS JR, CULVER DH, GAYNES RP. Nosocomial infections in medical intensive care units in the United States: National Nosocomial Infections Surveillance System. Crit Care Med. 1999;27:887-92.

4. HORAN T, CULBER D, JARVIS W. Páthogens causing nosocomial infections. Antimicrob. Newsletter 5 65-7. 1988.

5. SOCIEDADE BRASILEIRA DE PNEUMOLOGIA E TISIOLOGIA et al. III Consenso Brasileiro de Ventilação Mecânica. J Bras Pneumol, v. 33, n. Suppl 2, p. S1-S150, 2007.

6. SARMENTO, George Jerre Vieira (Ed.); VEGA, Joaquim Minuzzo (Ed.); LOPES, Newton Sergio (Ed.). Fisioterapia em UTI: avaliação e procedimentos. São Paulo: Atheneu, 2006. v. 1. 353 p.

7. FISCHER H, WIDDICOMBE JH. Mechanisms of acid and base secretion by the airway epith lium. $J$ Membr Biol 2006; 21(3):139-50. 
8. LOPES.A.J; NORONHA.A.J, MARFORT.T.T Mecanismo de defesa do aparelho Respiratorio. Revista do Hospital Universitário Pedro Ernesto.UERJ. 2011. National Nosocomial Infections Surveillance (NNIS) System.National Nosocomial Infections Surveillance (NNIS) System report, data summary from January 1990-May 1999, issued June 1999. Am J Infect Control. 1999;27:520-32.

9. SIBILLE Y, REYNOLDS HY.Macrophages and polymorphonuclear neutrophils in lung defense and injury.Am Rev Respir Dis.1990; 141(2):471-501.

10. TRINDADE SHK, MELLO - JUNIOR JF,MIOR OG, LORENZI-FILHO G,MACHIONE M, GUIMARÃES EL, SALDIVA PHN. Metódos do estudo do transporte mucociliar. Rev Bras Otorrinolaringol.2007;73(5):704-12.

11. ZHANG P.SUMMER WR, BABY GJ, Nelson.Innate immunity and pulmonary host defense.Immunol Rev.200;173:39-51

12. DAVID C. M. Ventilação mecânica: da fisiologia à prática clínica. Rio de Janeiro: Revinter, 2001 cap 5, p 63-72. Dispositivos Rathgeber J. usado para umidificar gases respiratórios. respir care clin n am. 2006; 12:165-82.

13. SCANLAN CL, WILKINS RL, STOLLER JK. Fundamentos da terapia respiratório de EGAN. $7^{\circ}$ ed. Ed. Manole, 2000; 31: 683-703.

14. CARVALHO, W. B. Atualização em Ventilação Pulmonar Mecânica. Atheneu. São Paulo, 1997.

15. SOTTIAUX,TM, consequences of under- and over-humidification, respir care clin $n$ am. 2006,12 (2): 233-52.

16. SIQUEIRA, T. B., COSTA, J. C. G. F., TAVARES, I. C., TORRES, P. M., ANDRADE, M. A., FRANÇA, E. É. T., FILHO, V. C. G., ANDRADE, F. M. D. Mecânica respiratória de pacientes neurocríticos sob ventilação mecânica submetidos à umidificação aquosa aquecida e a um modelo de filtro trocador de calor. Revista Brasileira Terapia Intensiva. 2010; 22 (3):264-269.

17. RICARD JD, LE MIERE E, MARKOWICZ P, LASRY S, SAUMON G, DJEDAIINI K. Efficiency and safety of mechanical ventilation with a heat and moisture exchanger changed only once a week. Am J Respir Crit Care Med. 2000;161(1):104-9.

18. RIBEIRO,D.C.,LOPES, C. R ., LIMA, R. Z.,TEIXEIRA ,Y . N. Comparison of the resistance imposed by artificial filters during mechanical ventilation.Arq Med ABC 32 (Supl. 2):S42-6.

19. AARC diretriz de prática clínica. Umidificação durante a ventilação mecânica. Associação Americana para Respiratory Care. Cuidados Respir. 1992; 37:887-90.

20. BOISSON C VIVIAND X, ARNAUD, L THOMACHOT, MILIANI Y, C MARTIN. Changing a hydrophobic heat and moisture exchanger after 48 hours rather than 24 hours: a clinical and microbiological evaluation. Intensive Care Med. 1999.

21. THOMACHOT L, BOISSON C, ARNAUD S, MICHELET.T CAMBOM S, MARTIN C. Changing heat and moisture exchangers after 96 hours rather than after 24 hours: a clinical and microbiological evaluation. Crit Care Med. 2000.

22. VINCENT J.L, BIHARI DJ, SUTER PM, BRUINING HA, WHITE J,NICOLAS-CHANOIN MH, et al. The prevalence of nosocomial infection in intensive care units in Europe. Results ofthe European Prevalence of Infection in Intensive Care(EPIC) Study. EPIC International Advisory Committee.JAMA. 1995;274:639-44.

23. SILVÉRIO, R. C., TAVARES, K. F., LACERDA, C. R., CARNEIRO, E. D., Incidência de Complicações Pulmonares em Pacientes Submetidos aos Sistemas de Umidificação Sob Ventilação Mecânica. Revista Triângulo: Ensino, Pesquisa e Extensão Uberaba - MG, v.1. n.1, p. 111-119, jul./dez. 2008. 
24. CARRILHO, C. M. D. M., GRION, C. M. C., CARVALHO, L. M., GRION, A. S., MATSUO, T. Pneumonia Associada à Ventilação Mecânica em Unidade de terapia intensiva Cirúrgica. Revista Brasileira Terapia Intensiva Volume 18 - Número 1 - Janeiro/Março 2006.

25. CHASTRE, J. Conference Summary: Ventilator-Associated Pneumonia. Respiratory Care. July 2005 Vol 50 Numero 7.

26. BOOTS, ROBERT J. MB BS, FRACP; HOWE, SHARRON ATCOP; GEORGE, NARELLE MSC; HARRIS, FIONA M. MB. Clinical utility of hygroscopic heat and moisture exchangers in intensive care patients. Crit Care Med. 1997 Oct;25(10):1707-12.

27. GUIMARÃES M. M. Q; ROCCO J.R. Prevalência e prognóstico dos pacientes com pneumonia associada à ventilação mecânica em um hospital universitário. Jornal Brasileiro de Pneumologia 2006; 32(4):339-46. 